Communications in Physics, Vol. 20, No. 3 (2010), pp. 255-264

\title{
FIRST PRINCIPLE STUDY ON AIN NANO WIRE
}

\author{
VU NGOC TUOC AND NGUYEN VIET MINH \\ Institute of Engineering Physics, Hanoi University of Technology
}

\begin{abstract}
We present a first-principle study on the atomic and electronic structure of AlN Nanowire and examine the dependence of surface stress on nanowire lateral size and shape. The hexagonal wire size ranging as 10-16-22 $\AA$ and triangular wire ranging from 10-13-16-20A. We investigate the unsaturated dangling bond state in the region of bandgap with varying the wires diameter. We also calculated the surface formation energy and find that it decreases with increasing the wire diameter and a greater stability (lower surface formation energy) comes with hexagonal wires. We also study the dependence of theoretical prediction on various density functional theory (DFT) treatment using Dmol3 local orbital density functional method with an effective core potential.
\end{abstract}

\section{INTRODUCTION}

Semiconductor nanowires [1] are attractive building blocks in nanotechnology applications for nanoscale electronic devices, including field-effect transistors (FETs), inverters, logic circuits, and decoders, because of the intrinsic small size and promise of enhanced mobility from 1D confinement effects. There has been the continued progress toward integrated nanoelectronic circuits which require advances in ability to better control the electronic properties of these building blocks and to assemble them into increasingly complex structures. AlN (as well as other heterostructure compound III-V Aluminum nitride based) is the wide band gap semiconductor with a band gap of $6.2 \mathrm{eV}$ and exhibits good dielectric properties, high thermal conductivity, and low thermal expansion coefficient. It also has potentials for application in deep ultraviolet optoelectronics and spintronics. Moreover very recently it is exposed that quasi 1D nanostructures of these material are the potential Hydrogen Storage Material and Solar Cells applications. Hydrogen, the third most abundant element on the earth, has the potential to meet the energy needs of the future. However, its economical use as an alternate energy has substantial difficulties to overcome. Among these, the most difficult challenge is to find materials that can store hydrogen with large gravimetric and volumetric density (6-10 wt\%) and operate under ambient thermodynamic condition, i.e. reversible adsorbtion/desorbtion $\mathrm{H}_{2}$ in the temperature range of $20^{\circ} \mathrm{C}$ to $50^{\circ} \mathrm{C}$ and under moderate pressures (max. $100 \mathrm{~atm}$ ). The first requirement limits the choice of storage materials to be composed of elements lighter than $\mathrm{Al}$, while the latter requires hydrogen binding energies to be between physisorption and chemisorption energies, i.e. optimal adsorption energy for $\mathrm{H}_{2}$ should be in the range of 0.1-0.2 eV $/ \mathrm{H}_{2}$. Unfortunately, the above two requirements are difficult to satisfy simultaneously. The bonding of hydrogen in light elements is either too strong, as in light metal hydrides and organic molecules, or too weak, when interacting with graphite and 
carbon/BN fullerenes and nanotubes. The challenge, therefore, has been to find systems where clustering of metal atoms can be prevented without compromising the binding energy of hydrogen. Recent efforts have focused on exposed light metal sites such as $\mathrm{Al}_{3}$, $\mathrm{Mg}_{2}$, and Li in light material substrate. Also it is supposed that when Al ions are embedded in some appropriate matrices, the adsorption energy of hydrogen molecules can be tuned. It would be ideal if these metal centers form the intrinsic backbone of the storage material so that their clustering will not be an issue to deal with. The answer lies in the choice of AlN quasi 1D nanostructures such as nanowires, nanocages, nanohorns, and nanotubes. The present work represents an extensive study of AlN wurtzite systems, which we perform using first-principles Density Functional Theory calculations.

\section{METHODOLOGY}

DFT calculations are performed using $\mathrm{DMOL}^{3}$ code $[2,3]$ where we employ the generalized gradient approximation (GGA) of Perdew and Wang (PBE). Core electrons are represented by the semilocal density functional semicore pseudopotential (DSPP) [4] with the electron configurations of Aluminum $1 \mathrm{~s}^{2}, 2 \mathrm{~s}^{2}, 2 \mathrm{p}^{6} 3 \mathrm{~s}^{2} 3 \mathrm{p}^{1}$ and $3 \mathrm{~d}$ in Double numerical basis with polarization functions, i.e., functions with angular momentum the configuration higher than that of highest occupied orbital in free atom (DND scheme), nitrogen $1 \mathrm{~s}^{2}, 2 \mathrm{~s}^{2}$, $2 \mathrm{p}^{3}$, and hydrogen $1 \mathrm{~s}^{1}$ using a real-space cutoff of 9 bohr. For that the DFT energy gap is

(A)

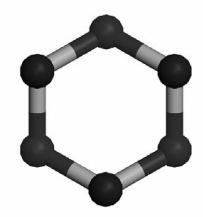

(B)

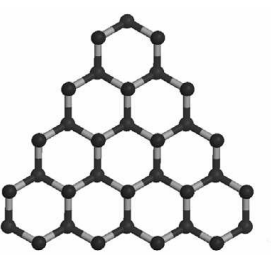

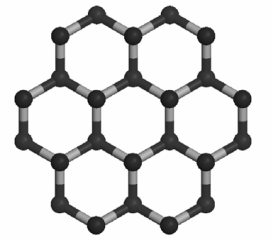

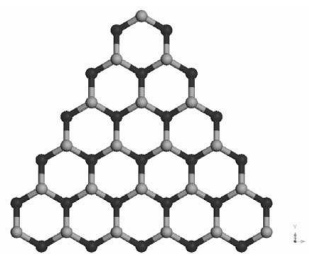

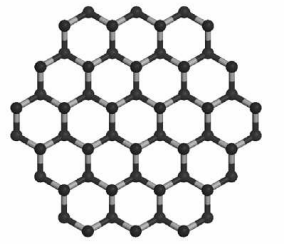
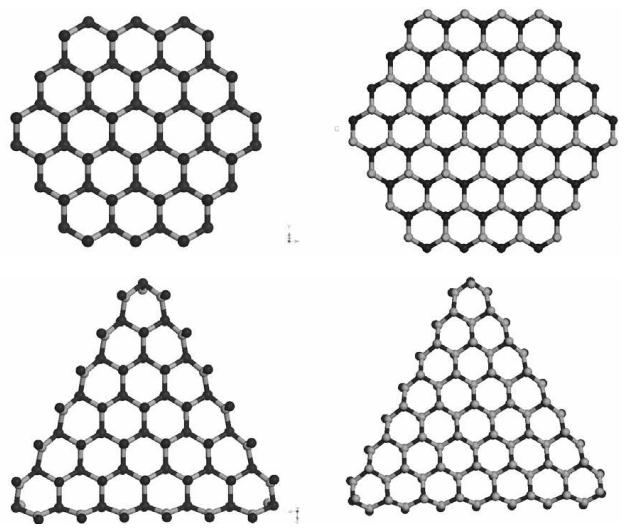

Fig. 1. (Color online) AlN nanowires in [0001] direction with (A) hexagonal and (B) triangular shaped cross sections, viewed along the wire direction. Nitrogen and Aluminum atoms are indicated by the light (purple) and dark (blue) spheres, respectively.

direct and equal $4.83 \mathrm{eV}$, and bulk optimized lattice parameters are $\mathrm{a}=3.131 \AA$, $\mathrm{c}=5.02 \AA$. Nanowires are sampled with a $1 \times 1 \times 4 \mathrm{k}$-grid. The effective diameter for both hexagonal and triangular shapes is considered as diameter of the circumsribed circle, which varies from approximately 10 to $31 \AA$. The shapes of nanowires are chosen in such a way as to minimize the number of dangling bonds on outer edge-layer atoms, that have a minimum coordination of 3 , leaving at most one dangling bond on the edge atoms. We examine the atomic structure and electronic properties of unsaturated as well as some hydrogen 
terminated wire. All unsaturated nanowires considered are shown in Fig. 1. From left to right the wires listed in Table 1 to have from 1-4 (hexagonal), and 4-7 (triangular) honeycomb block in each facet which are denoted as H-1 through H-4 and T-4 through T7 , respectively. The number of surface atoms and total number of atoms is very important characteristic for their ratio is directly related to surface-to-volume ratio also are listed here.

Table 1.

\begin{tabular}{|c|c|c|c|c|}
\hline "Hexagonal nanowire & 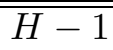 & 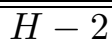 & 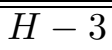 & 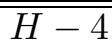 \\
\hline 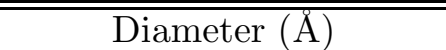 & 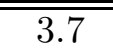 & 9.96 & $\overline{15.6}$ & 22.5 \\
\hline Number of Surface atoms & 12 & 36 & 60 & 84 \\
\hline Total number of atoms & 12 & 48 & 108 & 192 \\
\hline Triangular nanowire & $\bar{T} T-4$ & $\bar{T} T-5$ & $\overline{T T-6}$ & $\bar{T} T-7$ \\
\hline 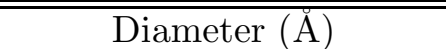 & 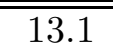 & 16.4 & $\overline{19.5}$ & 23.0 \\
\hline Number of Surface atoms & 48 & 60 & 72 & 84 \\
\hline Total number of atoms & 66 & 92 & 122 & 156 \\
\hline
\end{tabular}

\section{RESULTS AND DISCUSSION}

\section{III.1. Atomic structure}

By examining the nanowires with different shapes, when dangling bonds are not saturated then the surface relaxation in a nanowire occurs inevitably compare to that is taken from its stress-free bulk counterpart. This relaxation may initially deform the nanowire without any applied loads. Therefore, relaxation must be conducted in atomic simulations of nanowires to let them reach thermodynamics equilibrium state in both wires growth direction [0001] (wire axial) and its perpendicular surface. Similar result is obtained for all sized nanowires, so we have selected two representatives for nanowire. As a result the bond length at the outermost surface layer along hexagonal wire direction have changed ranging from 1.8 to $1.84 \AA$ (see Fig. 2). This behavior is found across the entire range of diameters and the shapes of nanowires examined. Compared to the bulk value of $1.94 \AA$, this represents a contraction of $5.1 \%$ to $7.0 \%$.

For the edge $A l-N$ pair: the Mulliken charge $+0.89 \mathrm{e}$ for $\mathrm{Al}$ and $-0.98 \mathrm{e}$ for $N$ at the corner edge (for both hexagonal and triangular shape) where as in the central core they are $1.26 \mathrm{e}$ and $-1.22 \mathrm{e}$ showing the redistribution of charge density due to the dangling bond at the outer most layer.

At the 1st inner layer from the surface of the nanowires, the contraction is less than $0.7 \%$ then within the second layer beneath the surface, it is less than $0.2 \%$. The other $A l-N$ bond which forms a zigzag chain from the outermost surface, running perpendicular to the wire direction, also be contracted during relaxation but in less extend which ranging from $2.5 \%$ to $5 . \%$ and its beneath layer is less than $0.9 \%$. These lengths stabilized and approaching the free surface value with increasing the wires diameter. 

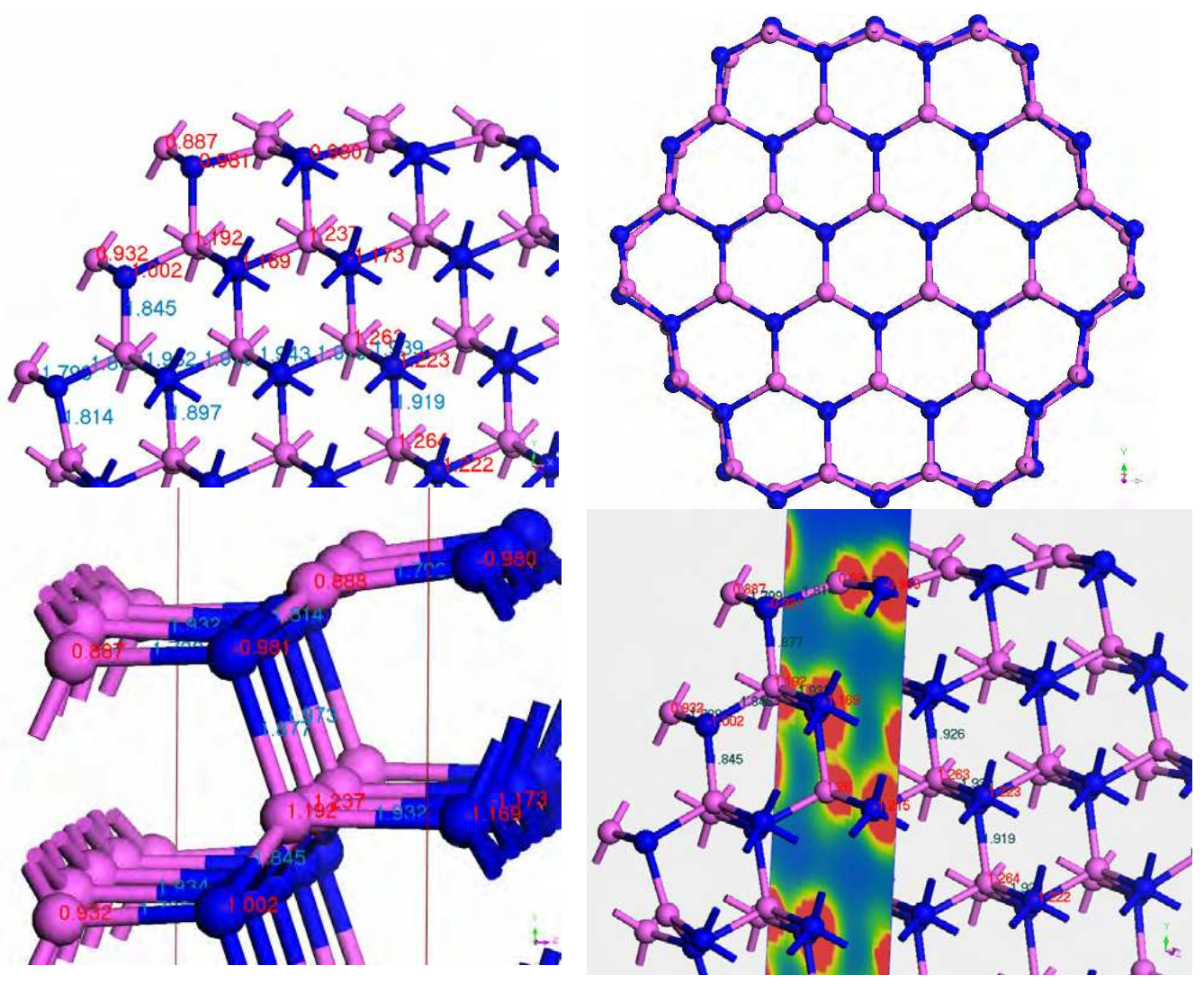

Fig. 2. (Color online) AlN Nanowires in [0001] direction with one representative hexagonal H-3 fully optimized structure with cross section view and a view along the wire direction. Nitrogen and Aluminum atoms are indicated by the light (purple) and dark (blue) spheres, respectively. The red label (lighter) is on the atom site indicated the Mulliken charge of the atom (showing the charge transfer within a $A l-N$ polar bond). The cyan lable (darker) in the midle $A l-N$ bond showing the length of the corresponding $A l-N$ bond. The cut plane showing electron density redistribution mapping between the atom $\mathrm{Al}$ and $N$ sites.

\section{III.2. Band structure and Density of State (DOS) analysis}

Band structures for relaxed nanowires are shown along the $\Gamma Z$ direction (parallel to wire direction) are shown in Fig. 5. The $1 \mathrm{D}$ band structures were computed using $1 \times 1 \times 4 k$-points. Similar result is obtained for all sized nanowires, so we have selected two representatives for nanowire. The band gap decreases with increasing nanowire diameter approaching bulk parameter. The band analysis show that the top of valence band does not change much for all the wires, but the bottom of the conduction band moves up as the diameter decreases, which results in the band gap becoming wider. It can be seen that, the bottom of conduction band and the top of valence band were both located in $\Gamma$ point of the Brillouin zone, indicating a direct band gap semiconductor . 

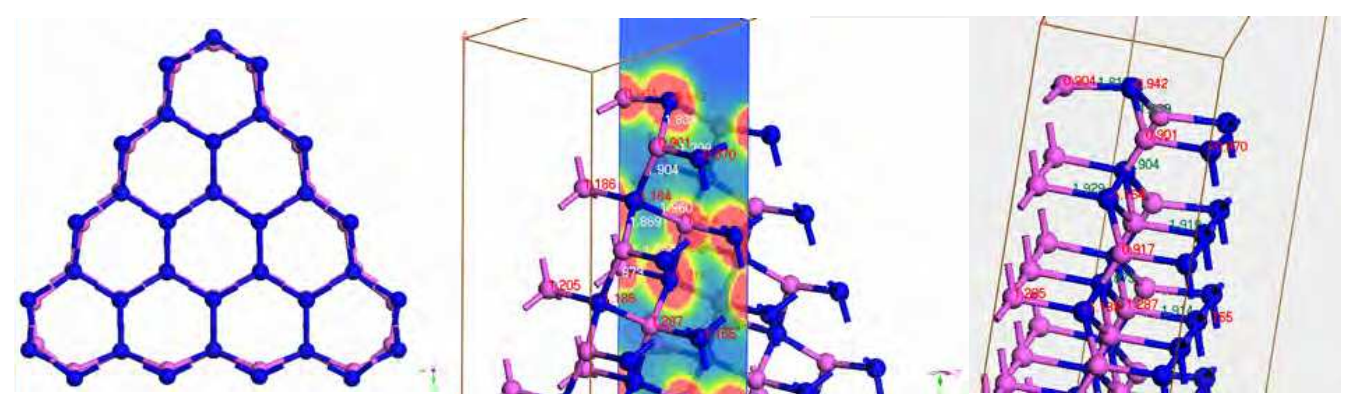

Fig. 3. (Color online) AlN Nanowires in [0001] direction with one representative triangular (T-4) fully optimized structure with cross section view and a view along the wire direction. See Fig. 2 for details.

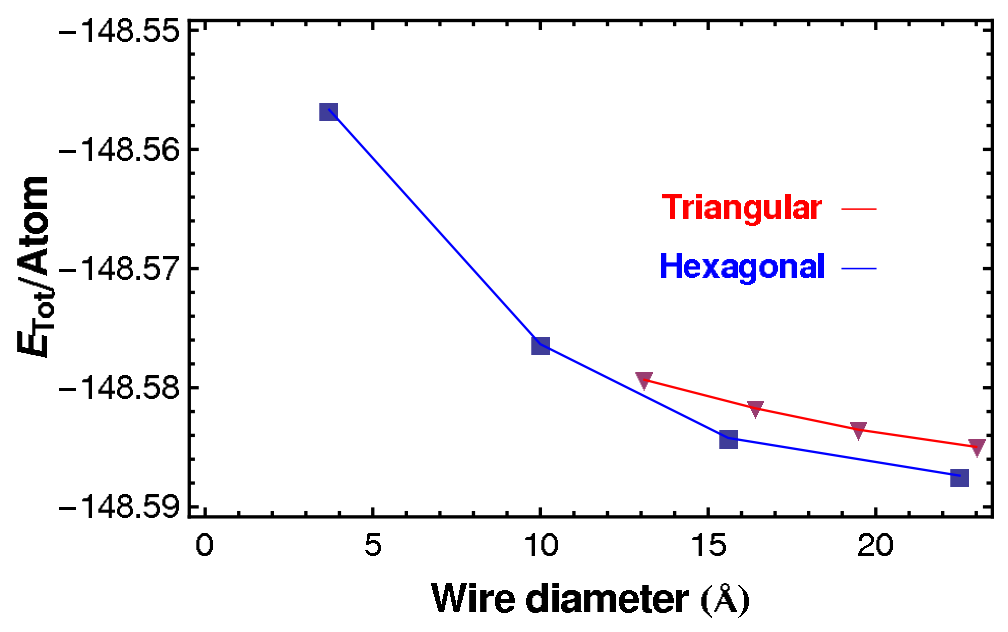

Fig. 4. (Color online) Diameter dependence of the cohersive energy for the various type AlN nanowire. The blue line (dark) with filled box is hexagonal and purple (light and dashed) with triangular is triangular wire, respectively.

The band gaps of triangular nanowires are smaller than for hexagonal wires with similar diameter and this difference is decreasing with diameter. Although this may due to our definition of the effective wire diameter, the triangular and hexagonal nanowires contain different numbers of AlN units (see Table 1) and smaller for triangular, so when we examine the band gap as a function of the number of atoms, the band gaps of triangular nanowires will go closer the gap of hexagonal and approaching the bulk value together. This trend can be explained as the triangular wires are less stable, and the occupied edgeinduced states are at a higher energy compared to the hexagonal one thus the formation energy is higher i.e., less stable (see more in Fig. 7). These results are in good accordance with other authors result, e.g. H. Xu et.al [5] as well as J. Li et al. [6]. This implies the clear dependence between the nanowire diameter and its resulting band gap, so that wire diameter can be used as one parameter to tailor the bandgap for a particular device application. Although it should of course be taken into consideration that band gaps 

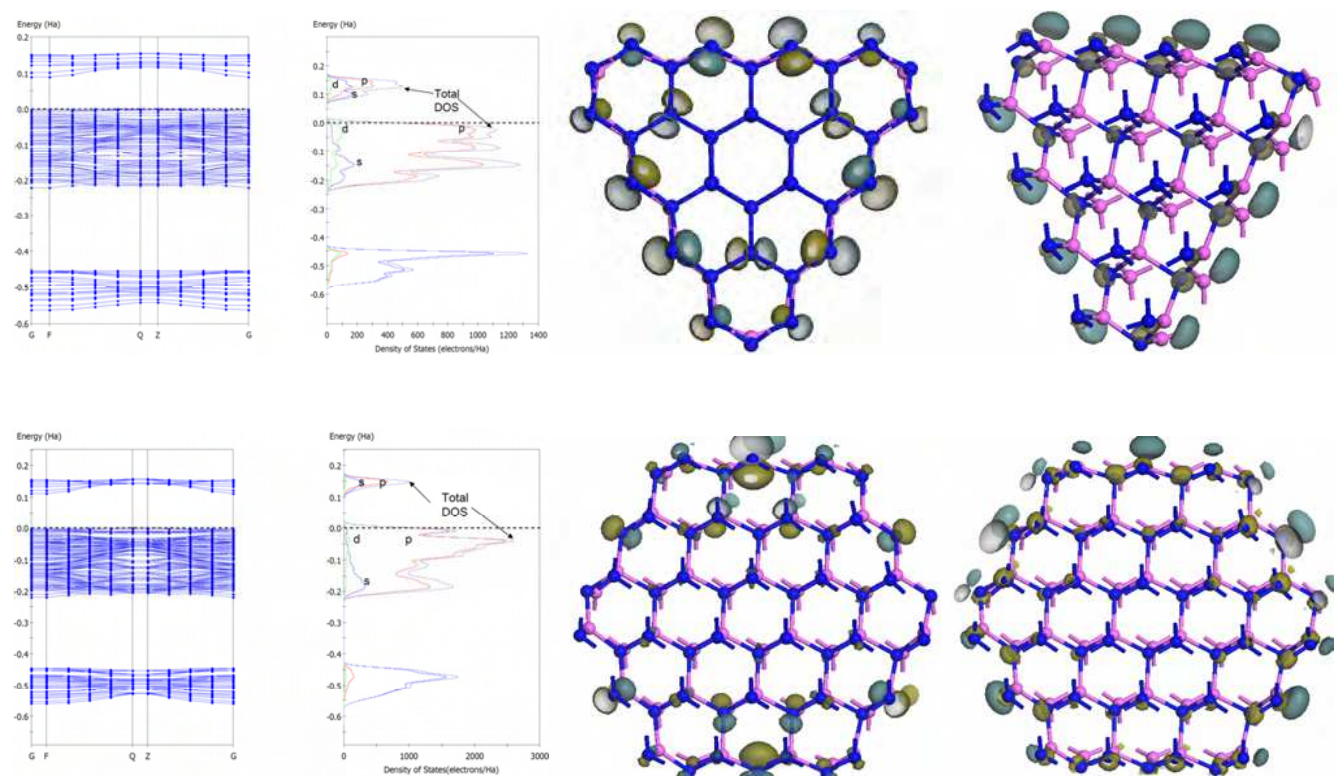

Fig. 5. (from the left to right) The band structures for relaxed triangular (T-4) and hexagonal (H-3) nanowire. The total DOS as well as PDOS of H-3 and T-4 are showing next to the bandstructures. The (HOMO) and (LUMO) states of the wire.

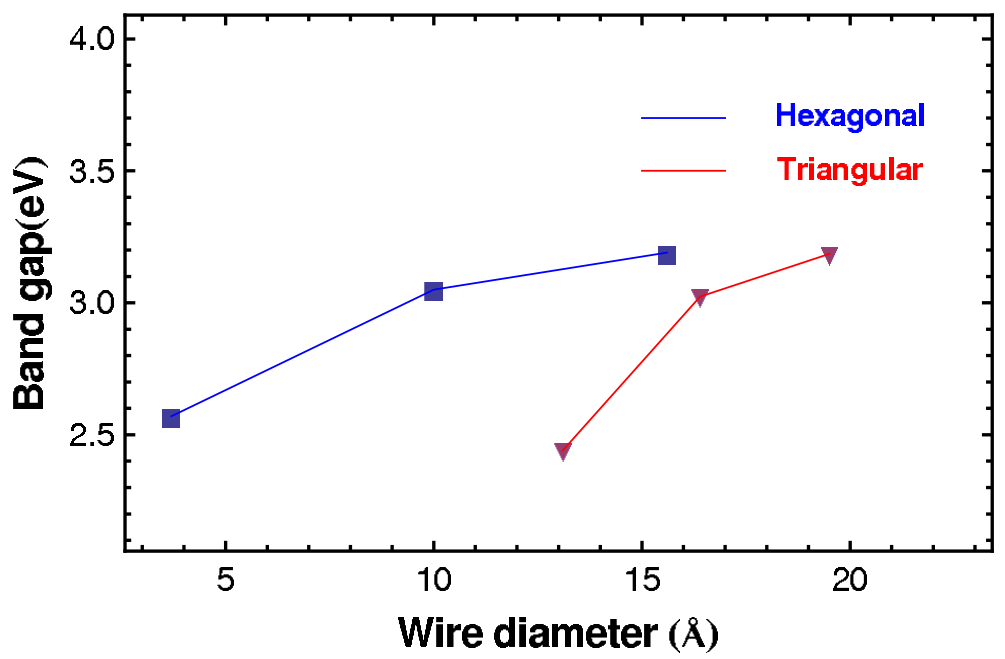

Fig. 6. Band gap as a function of nanowire diameter, filled box for hexagonal and triangular for triangular wire, respectively.

calculated using DFT are systematically underestimated but the trend is reserved. The Density of State (DOS) of the two representative wires have shown in Fig. $5 \mathrm{H}-3$ and T-4 showing that the Conduction Band Minimum (CBM) is mainly contribution of the 
$s$-orbital whereas the Valence Band Maximum (VBM) is $p$-orbital and this trend is for all wires. Looking closely at the highest occupied molecular orbital (HOMO) and the lowest unoccupied molecular orbital (LUMO) states in Fig. 5, we can see that relaxed nanowires have a HOMO orbitals localized on the reconstructed outermost layer dominated on Nitrogen size with $p$-character whereas the LUMO only has significant weight at the outermost layer located in both the Al site (more noticeable) with $s$ - character and also $N$ site (less noticeable part) also with $p$-character.

\section{III.3. Surface Formation Energy}

The surface relaxation is a process of energy minimization, in which atoms rearrange their positions to lower the total energy leading to a change in the lattice spacing of a nanowire it also has been observed in our simulations. The surface formation energy for one free surface is generally defined as

$$
E_{\text {form }}^{2 D}=\left(E_{t o t}-n_{N-a t} \mu_{N_{2}}-n_{A l-a t} \mu_{A l}\right) / A
$$

where $E_{\text {tot }}$ is total energy of the relaxed surface $n$ is number of each atom type, $\mu$ is chemical potential of the of atom species involved and $A$ is surface area. For the small structure as the our wire case the surface area is ill-defined and so we will change by the number of surface atoms. Thus the formation energy for the wire will be as:

$$
E_{\text {surf.form }}^{\text {wire }}=\left(E_{\text {wire }}-x . E_{\text {bulk }}\right) / N_{\text {surf.atom }},
$$

where $E_{\text {wire }}$ is total energy of the unsaturated wire, $E_{\text {bulk }}$ is bulk energy and $x$ number of the stoichiometric unit and $N_{\text {surf.atom }}$ is number of the total surface atoms.

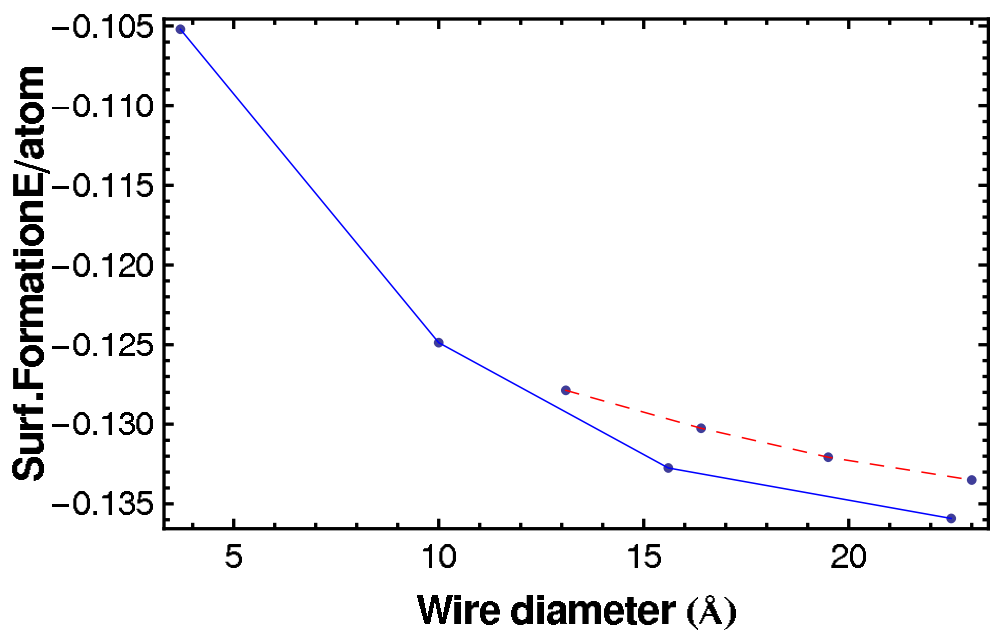

Fig. 7. (Color online) The wire surface formation energy is calculated by (2) with solid blue line (dark) with filled box for hexagonal and purple (light and dashed) with triangular for triangular wires, respectively.

The similar trend to above result is again observed as decreasing with increasing wire diameter and more stability, i.e. lower surface formation energy, come with the hexagonal wires. 


\section{III.4. Hydrogen storage problem}

In contrast to bulk AlN, which has the wurtzite structure and four-fold coordination, the $\mathrm{Al}$ sites in $\mathrm{AlN}$ low dimensional nanostructures are unsaturated and have three-fold coordination. So that each $\mathrm{Al}$ atom is capable of binding one $\mathrm{H}_{2}$ molecule in quasi-molecular form, leading to $4.7 \mathrm{wt} \%$ hydrogen, irrespective of the topology of the nanostructures. The binding energies of hydrogen to these unsaturated metal sites lie in the range of 0.1-0.2 $\mathrm{eV} / \mathrm{H}_{2}$ and are ideal for applications under ambient thermodynamic conditions. Furthermore, these materials do not suffer from the clustering problem that often plagues metal-coated carbon nanostructures $[7,8]$. Unlike carbon, which forms a planar graphite structure as its ground state, AlN does not form a layered structure. However, AlN, similar to that of $C$, exists in the form of nanocones, nanotubes, and nanowires, although the electronic structure of AlN nanocages is very different from that of $C_{60}$, where threefold $C$ atoms are stabilized by a rather strong bonding, which is nearly not the case for Al. AlN nanostructures have been successfully synthesized from the nonlayered structure. On the surface of these nanostructures, Al ion is two- or three-fold. When going from the four-fold coordinated bulk phase to the two- or three-fold coordinated nanostructures surfaces, unsaturated $\mathrm{Al}$ sites are naturally introduced, carrying a positive charge and provide the desirable adsorption sites for $\mathrm{H}_{2}$ molecules. Moreover, motivated by the success of the so-called "epitaxial casting" technology [1] to produce the nanotuble-like structure of non-planar nanomaterial for promising application in nanodevice, we also have studied the tube-like structure created from hexagonal nanowire with taken core and characterized by number of honeycomb wall layer (equivalent to two atomics layers)[9]. The adsorption energies for hydrogen atoms and molecules can be derived from total energy computation and defined as [10]

$$
E_{\text {adsorpt }}=\left(E_{\text {wire }}^{\text {sat }}-E_{\text {wire }}^{\text {unsat }}-n_{H}^{a t / m o l} \cdot E_{H}\right) / n,
$$

where, $E_{\text {wire }}^{\text {sat }}$, $E_{\text {wire }}^{\text {unsat }}$ and $E_{H}$ are the total energy of the hydrogen-saturated AlN tubelike, the bare tube and the hydrogen atom or molecule, respectively, and $n$ stands for the number of adsorbed hydrogen atoms or molecules. The negative value of $E_{\text {wire }}^{\text {sat }}$ corresponds to exothermic adsorption reaction and leads to stable state towards dissociation into AlN nanowires and hydrogen.

For the case of atom hydrogen adsoprtion (at both outer and inner three-fold surfaces atoms) corresponding to the case of chemisorbtion (chemical bonding or atomic adsorption), the Al-H length is in average 1.602 $\AA$ and the Mulliken charge of hydrogen atom is $-0.178 \mathrm{e}$ and the adsorption energy per atom is $1.2 \mathrm{eV}$. For Nitrogen site the $\mathrm{N}-\mathrm{H}$ is shorten $1.028 \AA$ and hydrogen charge $+0.375 \mathrm{e}$, i.e. much more charge transfer to Nitrogen site so that the adsorption energy per atom is higher as $1.9 \mathrm{eV}$ compare to $23 \mathrm{eV}$ in carbon materials. For the case of molecule hydrogen adsorption corresponding the physisorption or molecular adsorption, i.e. Van der Waals (vdW) with binding energies is an order less than that of chemisorbtion, the distance from $\mathrm{Al}$ to $\mathrm{H}_{2}$ is $2.43 \AA$ and $H-H$ bond length is $0.775 \AA$ (showing at the last image in Fig. 8) corresponding the adsorprion energy per molecule is $0.2 \mathrm{eV}$ and $\sim 4 \mathrm{wt} \%$ storage capacity of molecular hydrogen which is suitable to the range of technological application. 

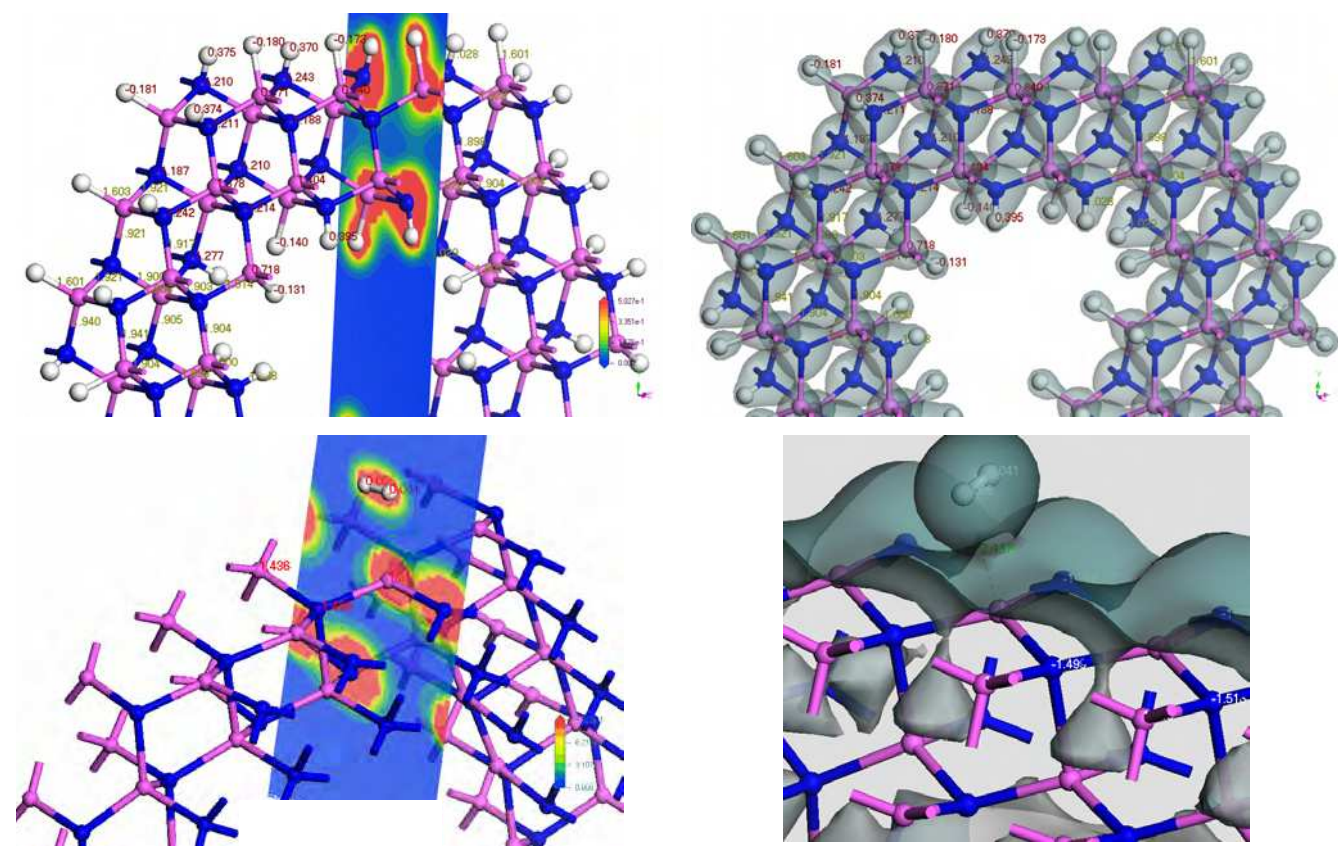

Fig. 8. ((upper row) Relaxed hexagonal tube-like nanowire terminated by hydrogen atom $(\mathrm{Al}-\mathrm{H})$ and $(\mathrm{N}-\mathrm{H})$ from left to right with i) Mulliken charge labeled in atom site and bond length label in selective bonds and cut plane showing charge redistribution around the bond; (ii) isosurface with charge density with selective Mulliken charge and bond length; (lower row) Relaxed hexagonal tube-like nanowire terminated by hydrogen molecule $\left(\mathrm{Al}-\mathrm{H}_{2}\right)$ with similar detail as in the upper row.

In summary, we have examined the atomistic and electronic structures and stability of nanowire (hexagonal and triangular shapes) and hydrogenated nanotube-like of wurzite AlN grown in [0001] direction of a wide range of diameters 4-23A. The surface relaxations causes the bond strained in length and angle similarly for all studied wires with the strain energy decreasing with increasing wire diameter for all shapes of wires. Quantum confinement effect, expressed here via the wire diameter and the surface relaxation are the two factors that dominate the influence on all wires characteristics such as bandgap, surface formation energy, DOS and PDOS. Our simulation results agree well with other theoretical and experimental data.

\section{ACKNOWLEDGMENT}

This work was supported by the Vietnamese NAFOSTED program No. 103.02.100.09.

\section{REFERENCES}

[1] Matt Law, Joshua Goldberger, and Peidong Yang, Annual Review of Materials Research 34 (2004) 83-122.

[2] B. Delley, J. Chem. Phys. 113 (2000) 7756. 
[3] B. Delley, Phys. Rev. B66 (2002) 155125.

[4] Damien J. Carter, Julian D Gale, Bernard Delley, and Catherine Stampfl, Phys. Rev. B77 (2008) 15349.

[5] H. Xu, A. L. Rosa, Th. Frauenheim, R. Q. Zhang, and S. T. Lee, Applied Phys. Lett. 91 (2007) 31914.

[6] J. Li and L. W. Wang, Phys. Rev. B 72 (2005) 125325.

[7] M. Zhao, Y. Xia, X. Liu, Z. Tan, B. Huang, C. Song, and L. Mei, J. Phys. Chem. B110 (2006) 8764.

[8] Q. Wang, Q. Sun, P. Jena, and Y. Kawazoe, Appl. Phys. Lett. 94 (2009) 13108.

[9] Q. Wang, Q. Sun, P. Jena, and Y. Kawazoe, ACS Nano, Article ASAP to be published 2009

[10] R. Z. Rajendra and I. D. Brett, Phys. Rev. B72 (2005) 045439.

Received 30 September 2009. 